Books, videos, CD-ROMs, DVDs and any other relevant items submitted for a review in the $B D J$ should be addressed to: Kate Maynard, Assistant Editor, British Dental Journal, NPG, 4-6 Crinan Street, London N1 9XW

\section{Goldfingered: Gold alloy and metal- ceramic restorations in dental practice (CD-ROM)}

P.B. Robinson
UK: The Dental Channel
price $€ 199.99$ including 12 hours of
verifiable CPD

This CD-ROM is produced by the dental channel (www.dental-channel.co.uk) with funding from the Department of Health. It is a highly interactive program that is encyclopaedic in detail and generally well illustrated. It aims to describe the various types of gold alloy restorations, revise the principles of their associated tooth preparations, consider the implications of adhesive technology upon these and review shade prescription, impression taking and provisional restorations.

There are four sections to the program: the core information/reference section; a self test quiz marked using negative scoring; informative links to further information sources; and a series of case studies. To access the full program, other than the reference material, the user must be both online and registered. A general dental services NHS contract number permits free registration and enables verifiable CPD hours to be accrued. Video clips are used extensively throughout the program and key terms may be clicked on to obtain further explanatory comments. Some terms, however, when clicked do not respond in this way - as was obviously intended - but these are very few in number and do not distract from this excellent program.

The incorporation of a useful notepad facility allows the user to write notes as they learn. There is also a helpful audio commentary that may be omitted if found to be distracting. Although a laudable attempt is made to link clinical practice to published research by the inclusion of 66 references to scientific papers, it is not clear how these papers were selected. The facility to click on the reference and bring up the paper's abstract is, on the other hand, very useful especially as potential users may not have ready access to library facilities.

Despite its funding it is refreshing to see that the content has not been constrained by the NHS Terms and Condition of Service - a comprehensive section on the gold foil technique is included! Although many techniques are updated with the application of adhesive technology it is pleasing to see that great stress is still placed upon the fundamentals of tooth preparation. To totally depart from this risks the loss of core technical skills in future dentists.

With such a complex array of topics and options it would be all too easy to lose one's way and forget what you have covered. The programers have thought of this too and a record of topics visited is automatically maintained by the software. Perhaps a minor quibble would be the rather complex list of instructions for loading the program. I would assume this is inevitable due to the wide ranging types of operating platforms on which the software has to be capable of running.

In light of the many good and innovative points of this program I would recommend this CD-ROM as an excellent source of CPD for interested practitioners.

R.G. Chadwick

\section{Oral hard tissue diseases: A reference manual for radiographic diagnosis}

\section{J.R. Newland \\ USA: Lexi-Comp \\ price $\$ 44.95$, pp 107 \\ ISBN 1591950287}

This is an excellent reference book for aiding in the radiological differential diagnosis of lesions of the jaws. It will be of particular benefit to those with less experience in diagnosis of jaw lesions.

The book is well laid out with 12 tabbed divisions based on the site, outline and lucency of lesions. Each section lists both common and uncommon conditions giving rise to the radiological appearance, followed by further information on the individual lesions which may aid differential diagnosis. The radiological features included are not extensive and do not compare to some of the textbooks available on dental radiology. The images contained within the book are of good quality, each condition illustrated by at least one and sometimes two radiographs. Other useful clinical information is also included which may aid definitive diagnosis, as well as a section on treatment, follow up and clinical significance.

Overall I think this is a very useful addition to a dentist's library and will also be valuable to general radiologists who seldom come across lesions of the jaws.

J. Luker

\section{Medical problems in dentistry}

\section{Scully, R.A. Cawson \\ UK: Elsevier \\ price Ł59.99, pp683 \\ ISBN 0443101450}

Every generation produces its classic texts; this is one. Such books tend to become larger with each edition, and this is no exception. Medical problems in dentistry was never a textbook in the standard sense; it was always a ready reference when one was unsure of the current dental management of a medical condition. As such it has become the standby of many clinicians. A copy sits in my clinic, not just for students and junior staff but for my own consultation, and it has become an invaluable aide and a first point of reference over the years. 

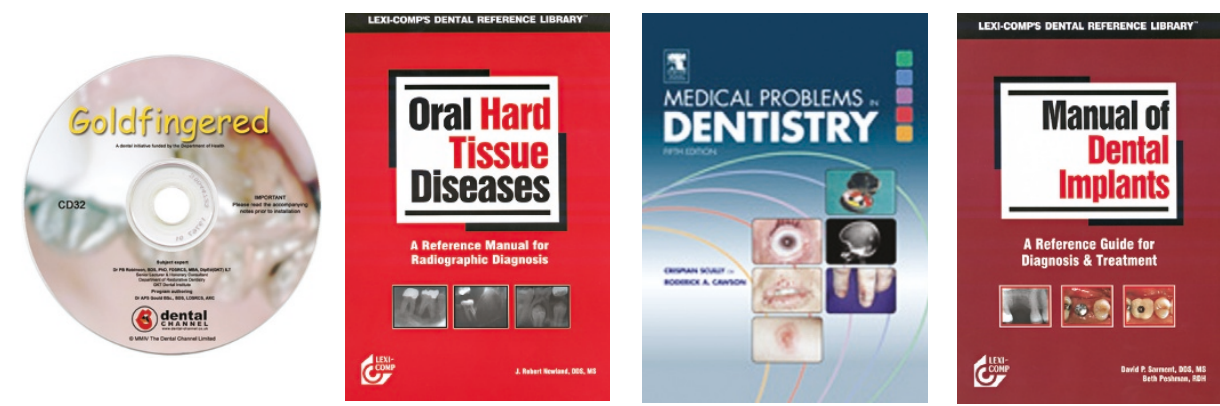

The scope of the text has widened greatly both with new sections (such as disability and complementary medicine) and by the strengthening of existing sections. For example, five pages are given to infections prevalent in the developing world (relevant in the age of mass worldwide tourism) and six pages to the medical problems encountered in different immigrant groups.

As the authors themselves state, this never purported to be a comprehensive textbook of medicine for dentists. The suggested reading lists go a long way towards making up for this and now include a number of trustworthy websites.

This is the first edition of Medical problems in dentistry with colour illustrations. Many clinical texts are spoiled by poor colour reproduction. Here the photographs are well chosen and of high quality; the authors clearly have access to a comprehensive collection of illustrations.

The appearance of the printed pages is a little daunting with dense pages of black type relieved by the occasional table or picture. However, when one begins to read, the logic of this layout becomes apparent; a lot of information is packed into a relatively small number of lines. The summary tables,

highlighted in colour, also make it easy to assimilate the relevant information.

It is difficult to know the target audience for this book. Is it aimed at a UK audience or the wider English speaking dental world? The relentless use of NHS derived forms as illustrations suggests the former; the authors may not have realised that some items, such as the chart of the British manual alphabet ('finger spelling') in the section on deafness, will be of little assistance to practitioners in other English speaking countries such as Australia, the USA and Ireland.

None of these criticisms are meant to detract from this superb book. Every dentist needs a copy near to hand. Dentistry already owes Crispian Scully and Roderick Cawson an immense debt for their various texts but this new edition of Medical problems in dentistry must stand as their greatest achievement to date.

B. McCartan

\section{Manual of dental implants: a reference guide for diagnosis and treatment}

\section{D.P. Sarment, B. Peshman \\ USA: Lexi-Comp \\ price $\$ 54.95$, pp 101 \\ ISBN 1591950619}

According to the introduction '...the Manual of dental implants is designed to initiate dental students, general dentists, dental hygienists and staff into the world of implant restorative dentistry and maintenance.' It aims to be a resource for dental professionals new to implant dentistry and as such it almost meets its aim. It concentrates on the restoration and maintenance of implants and it is not intended to be used by anyone intending to place implants. It is a spiral bound book, which adds nothing to its desirability but makes it practical to use as a reference whilst working.

The first of its six chapters is titled 'Basic principles' and is written in a style that appears to be aimed at the layperson rather than the professional. The next chapter on diagnosis is too brief for my liking, concentrating on radiographic investigations, although these are covered comprehensively.

Treatment planning is reasonably well covered in the next chapter but it is surprising that Tarnow's ${ }^{1}$ important study on the relationship between the interdental papillae and contact point in the aesthetic zone is not mentioned. The references listed in the extensive bibliography are not referred to in the text but divided into chapters. This would make life difficult for a student wishing to follow up any statements made in the text.

There are some interesting cases described in the chapter on restoration sequences with many excellent colour photographs, which are a useful aid to understanding the often complicated techniques.

Maintenance is allotted a chapter to itself, which is not surprising as one of the authors is a dental hygienist who incidentally is described as an employee of Straumann. It should be noted however that a number of different systems are described throughout the book with no particular emphasis on any one system.

The final chapter is titled 'Implants in practice' and is largely aimed at the US market, although the principles of involving the whole team in integrating implantology into a general practice should be considered by any dentist wanting to offer implants to their patients.

In general the text is written as a series of bullet points, which I found disjointed and difficult to follow in places. Many important issues in implantology are glossed over or not discussed at all and this is serious flaw in such a text.

\section{K. Gibney}

1. Tarnow D P, Magner A W, Fletcher P. The effect of the distance from the contact point to the crest of bone on the presence or absence of the interproximal dental papilla. J Periodontal 1992 63(12): 995-996. 